\title{
Alpha-helical cationic antimicrobial peptides: relationships of structure and function
}

\author{
Yibing Huang, Jinfeng Huang, Yuxin Chen (凶)
}

Key Laboratory for Molecular Enzymology and Engineering of Ministry of Education, Jilin University, Changchun 130021, China

$\bowtie$ Correspondence: chen_yuxin@jlu.edu.cn

Received September 22, 2009; accepted October 29, 2009

\begin{abstract}
Antimicrobial peptides (AMPs), with their extraordinary properties, such as broad-spectrum activity, rapid action and difficult development of resistance, have become promising molecules as new antibiotics. Despite their various mechanisms of action, the interaction of AMPs with the bacterial cell membrane is the key step for their mode of action. Moreover, it is generally accepted that the membrane is the primary target of most AMPs, and the interaction between AMPs and eukaryotic cell membranes (causing toxicity to host cells) limits their clinical application. Therefore, researchers are engaged in reforming or de novo designing AMPs as a 'singleedged sword' that contains high antimicrobial activity yet low cytotoxicity against eukaryotic cells. To improve the antimicrobial activity of AMPs, the relationship between the structure and function of AMPs has been rigorously pursued. In this review, we focus on the current knowledge of $\alpha$-helical cationic antimicrobial peptides, one of the most common types of AMPs in nature.
\end{abstract}

KEYWORDS antimicrobial peptides, mechanism of action, peptide structure, antimicrobial activity

\section{INTRODUCTION}

Antibiotic resistance has become a great concern in terms of public health, and the delayed development of new antibiotics to hinder growth of antibiotic resistance makes the problem more serious (Oyston et al., 2009). Therefore, new antimicrobial strategies and/or alternative drugs are required to revive the potency of traditional antibiotics and to guard human health (Marshall and Arenas, 2003; Marr et al., 2006).
Antimicrobial peptides (AMPs) represent such a new class of antibiotics. Many researchers have contributed to the understanding of the structure-activity relationship of AMPs (Ali et al., 2009; Joanne et al., 2009; Manna and Mukhopadhyay, 2009; Resende et al., 2009; Verdon et al., 2009). Traditional antibiotics generally target a particular physiological process of bacteria, such as cell wall synthesis, DNA replication etc. (Neu, 1992); however, due to mutations and adaptation in bacteria, the traditional antibiotics eventually become inefficient (Bryan, 1988). In contrast, most AMPs target the bacterial cell membrane without specific receptors, and thus, become an ideal approach to overcome the resistance resulting from bacterial mutations (Marr et al., 2006).

Nowadays, more than 1000 AMPs have been identified with antimicrobial activity (Wang et al., 2009). The general features of AMPs include: (1) Diversity; AMPs have been discovered in most species, from bacteria to mammals, in addition to designed, modified and synthesized AMPs. (2) Uniqueness; each AMP differs from others by unique size and sequence. (3) Secondary structure; AMPs are either present or fold into a category of secondary structures, such as $\alpha$-helix or $\beta$-sheet. (4) Physiological significance; AMPs not only exhibit the ability to kill or inhibit pathogenic microorganisms, but also play an important role in modulating the immune system in vivo. (5) Wide spectrum of killing activity; AMPs have been reported to kill or inhibit a variety of organisms or cells, including Gram-negative and Gram-positive bacteria, viruses, protozoa, parasites, fungi and even cancer cells (Marshall and Arenas, 2003; Jenssen et al., 2006).

Although AMPs are highly variable, most experts agree that AMPs are short peptides with 12-100 amino acids (Hancock, 1997; Jenssen et al., 2006). AMPs are positively charged, with net charge of $+2-+9$ due to the presence of 
basic amino acids (Lys, Arg). AMPs possess approximately $50 \%$ hydrophobic residues, which favors an amphipathic conformation upon interaction with membranes. As shown in Fig. 1, the secondary structure of AMPs include four major classes, $\alpha$-helix, $\beta$-sheet stabilized by two or three disulphide bridges, extended helices (polyprohelices) with a predominance of one or more amino acids and loop structures (Hancock, 1997; Jenssen et al., 2006).

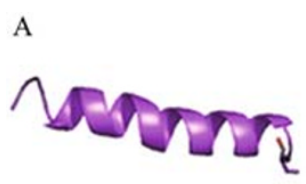

C

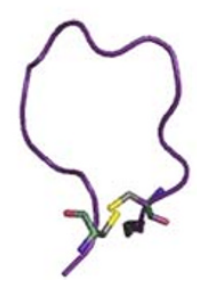

B

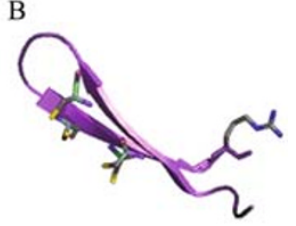

D

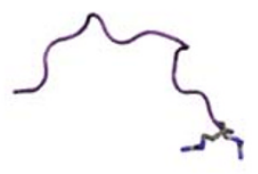

Figure 1. Molecular models of AMPs structures. These models are from the NMR structural database. (A) The amphipathic $\alpha$-helical structure of magainin 2 (PDB code 2MAG). (B) Two-stranded antiparallel $\beta$-sheet of protegrin 1 (PDB code 1PG1). (C) The $\beta$-turn loop structure of a cyclic indolicidin peptide derivative (PDB code 1QX9). (D) Structure of indolicidin bound to dodecylphosphocholine micelles shows the random coil structure (PDB code 1G89).

As one of most widely distributed AMPs, a-helical antimicrobial peptides (aAMPs) have been thoroughly investigated. Numerous studies have been performed to optimize their potential for clinical applications, i.e., to improve the antimicrobial activity and to reduce the toxicity against human cells. In this review, we will focus on the current knowledge about the structural-activity relationship of aAMPs exclusively in terms of antibacterial activity.

\section{MECHANISM OF ACTION}

The prokaryotic and eukaryotic cell membranes have different structures and functions. Inherent distinction in lipid composition provides a basis for the preference of AMPs toward a specific membrane, and provides researchers with an opportunity to find or design peptides with strong specificity. Prokaryotic membranes have a high negative net charge and are predominantly composed of phosphatidylglycerol (PG), cardiolipin (CL), or phosphatidylserine (PS). In contrast, mammalian membranes are enriched in the zwitterionic phospholipids (neutral net charge) phosphatidylethanolamine $(\mathrm{PE})$, phosphatidylcholine $(\mathrm{PC})$ or sphingomyelin (SM)
(Yeaman and Yount, 2003; Giuliani et al., 2007). Moreover, the mammalian cell membrane contains cholesterol, while the prokaryote membrane does not (Tytler et al., 1995). It has been reported that cholesterol can dramatically reduce the activity of AMPs by stabilizing the lipid bilayer or by directly interacting and neutralizing AMPs (Matsuzaki, 1999). Despite of the different composition, the difference on membrane asymmetry and transmembrane potential $(\Delta \psi)$ also provide alternatives for AMPs (Yeaman and Yount, 2003).

The mechanisms of action for AMPs are illustrated in Fig. 2. It is apparent that AMPs have various targets that eventually induce fatal damage to the pathogen. According to the distinct fatal targets, AMPs are categorized into two major groups, membrane disruptive AMPs and non-membrane disruptive AMPs (Powers and Hancock, 2003). Generally, the membrane disruptive peptides function through interaction with the cell membrane, permeabilizing the phospholipids bilayer and eventually causing cell death. This may result from lesions in the membrane barrier or by dissolving of the membrane through a detergent-like process. On the other hand, the nonmembrane disruptive peptides may pass through the membrane and interact with variable intracellular macromolecular targets through similar mechanisms as traditional antibiotics. Regardless of the precise mechanism, the interaction with the cell membrane is the key step for all AMPs (Hancock and Rozek, 2002).

For Gram-negative and Gram-positive bacteria, the bactericidal process of cationic AMPs is stepwise and generally rapid. Initially, driven by electrostatic forces, AMPs diffuse toward the cell membrane even from a relatively long distance (Mavri and Vogel, 1996). It is hypothesized that the negative charge on the outer bacterial envelope (the phosphate groups from Gram-negative bacteria, and the lipoteichoic acids from Gram-positive bacteria) (Yeaman and Yount, 2003; Jenssen et al., 2006) and the strong electrochemical gradient $(\Delta \psi)$ of the bacterial cytoplasmic membrane both contribute to the attraction of cationic AMPs (Yeaman and Yount, 2003). For Gram-negative bacteria, a mechanism termed "self-promoted uptake" has been suggested by Hancock (Hancock, 1997). After reaching the bacterial surface, cationic peptides competitively replace divalent cations due to different binding affinities. The divalent cations act as a stabilizer for the LPS by binding the anionic phosphate groups. The loss of $\mathrm{Mg}^{2+}$ and $\mathrm{Ca}^{2+}$ results in the disturbance of the rigid outer membrane and promotes the uptake of more molecules. During this process, many linear AMPs fold into an amphipathic structure due to the hydrophobic environment of the cell membrane. Consequently, the peptides contact the cytoplasmic membrane and disrupt the lipid bilayer in multiple ways. Initially, cationic peptides contact the negatively charged lipid head groups on the surface of the membrane, and an amphipathic conformation will be induced for most aAMPs. The amphipathic nature of AMPs is essential for further interaction with the hydrophobic components of the 


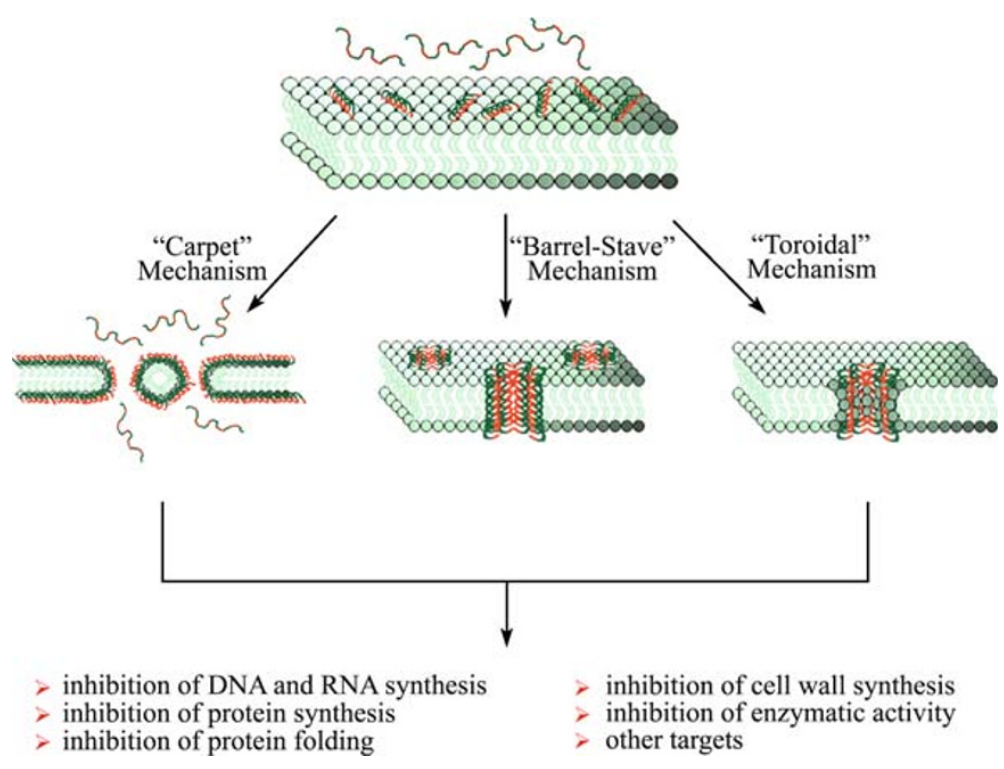

Figure 2. Models of interaction between cationic AMPs and cytoplasmic membrane. Cationic AMPs first associate with the negatively charged outer membrane surface in a parallel orientation. The models for the following membrane permebilization (membrane disruptive AMPs) include "Carpet" mechanism, "Barrel-Stave" mechanism and "Toroidal" mechanism.

membrane. During this folding process, it is proposed that AMPs adopt a parallel orientation to the membrane (Jenssen et al., 2006). Of the several models that attempt to explain the permeabilizing process, the carpet model, barrel-stave model and toroidal-pore model are widely accepted. An overview of the interaction of peptides with the bacterial cytoplasmic membrane is shown in Fig. 2.

In the carpet model (Pouny et al., 1992), the peptides initially associate with the membrane and align parallel to the surface of bilayer, covering the surface in a carpet-like fashion. This orientation destabilizes the packing of phospholipids and causes a change in membrane fluidity because of the displacement of phospholipids by peptides. Thus, the stability of the local membrane is disturbed. When a threshold peptide concentration is reached, the membrane will break down due to unfavorable energetics. The general features of the carpet model include that peptides remain in contact with the lipid head groups, they do not necessarily interact with the hydrophobic core of membrane, i.e., without insertion, the membrane is dissolved in a dispersion-like manner, and the disruption of membrane does not involve a channel-forming process (Powers and Hancock, 2003; Giuliani et al., 2007).

In the barrel-stave model (Ehrenstein and Lecar, 1977), peptides reorient perpendicularly to the surface of the membrane and are positioned as the "staves" in a "barrel"shaped aggregation, with the hydrophobic regions of peptides associating with the hydrophobic core of membrane, while the hydrophilic regions face each other and form an aqueous pore or channel. The formation of pore or channel is a dynamic process. Initially, peptides bind to the membrane surface, possibly as monomers. Then with a conformational phase transition, the hydrophobic regions of peptides insert into the membrane due to the interaction with complementary hydrophobic components of membrane. When a threshold peptide concentration is reached, peptide monomers selfaggregate through interactions between hydrophilic peptide amino acid residues leading to deeper insertion into the hydrophobic membrane core. Pores with variable sizes are dynamic structures formed by gaining or losing peptide monomers. There are three general features that define the barrel-stave model: (1) peptides make pores or channels through membrane in a perpendicular orientation, (2) membrane lipids are not involved in the inner face of pores and they do not bent or tilt together with peptides during the pore formation as comparison with the toroidal-pore model discussed below, and (3) the pore is a dynamic structure and well defined (Yeaman and Yount, 2003; Brogden, 2005; Giuliani et al., 2007).

In the toroidal-pore model (Matsuzaki et al., 1995, 1996), peptides first reorient with membrane lipid. Similar to the barrel-stave model, peptides insert perpendicularly to the membrane to form a pore, while the hydrophilic regions of the peptides keep the association with lipid head groups and the hydrophobic peptide regions associate with the hydrophobic core of the membrane lipids. Thus, the peptides and lipids together form well-defined pores, with the hydrophilic regions of the peptides and phospholipids head groups facing the center of the pore and producing an aqueous pore. With the tilt of lipid molecules, the membrane also curves inside to form a hole lined by the lipid head groups and covered by peptides (Yeaman and Yount, 2003; Giuliani et al., 2007). The toroidalpore model is supported by recent studies, which confirmed that these pores are formed by peptides, such as maganins, mellitin and LL-37 (Hallock et al., 2003; Wildman et al., 2003). 
The general features in toroidal-pore include that pores are constructed with peptides and lipids, peptides orient perpendicularly, and the pore-forming lipids change their positions relative to the bilayer membrane, from near perpendicularity to continuous angles.

Another mechanism, similar to the toroidal-pore model and known as the aggregate model, was first proposed by Wu et al. (Wu et al., 1999). In this model, AMPs reorient without particular orientation, insert into the membrane, and associate with lipids to form micelle-like complexes. The aggregated complexes form transient and transmembrane pores. This model can explain the translocation of peptide across the bilayer, and molecular dynamics (MD) simulations of the magainin MG-H2 peptide interacting with a model lipid membrane confirmed the aggregate model (Leontiadou et al., 2006). In contrast to the well-defined toroidal pore model, only one peptide is located in the center of the pore in the aggregate mechanism, and there is not a defined orientation for all peptides involved in pore formation.

Recently, based on the "barrel-stave" model (Ehrenstein and Lecar, 1977) and the "carpet" model (Shai, 1999), Chen et al. proposed a "membrane discrimination" model for AMPs whose sole target is the biomembrane (Fig. 3) (Chen et al., 2006, 2007). In this model, the peptide specificity to eukaryotic or prokaryotic cells depends upon the compositional difference in the lipids of membranes (Abraham et al., 2005). As mentioned above, eukaryotic cell membranes are characterized by the zwitterionic phospholipids that contain a relatively large amount of cholesterol and sphingomyelin, while prokaryotic membranes are distinguished by the negative transmembrane potential inside (Zilberstein et al., 1979; Lugtenberg and Van Alphen, 1983). For eukaryotic cells, peptides form pores/channels in the hydrophobic core of the bilayer membranes and cause the hemolysis of erythrocytes. For prokaryotic cells, the peptides lyse cells in a detergent-like mechanism, as described by the carpet model (Shai, 1999). The "membrane discrimination" mechanism agrees with the results of previous studies using model membranes. The pore formation mechanism ("barrel-stave" model) was used by AMPs in zwitterionic membranes, but the detergent-like mechanism ("carpet" model) was applied when the peptides interacted with negatively charged membranes (Ladokhin and White, 2001; Papo et al., 2002).

\section{STRUCTURE-ACTIVITY RELATIONSHIPS OF aAMPS AND THE IMPROVEMENT OF SELECTIVITY AND STABILITY}

The toxicity of AMPs against eukaryotic cells is the key obstacle for their clinical application. Use of erythrocyte hemolysis assays is a common approach in vitro to evaluate the toxicity of AMPs to eukaryotic cells and this assay also represents a convenient and rapid method to screen other molecules. For a given peptide, the hemolytic activity is represented by the maximum peptide concentration with no hemolysis ( $\mathrm{MHC})$. In contrast, the antimicrobial activity is represented by the minimum peptide concentration that inhibits bacterial growth (MIC), as determined by either microbroth serial dilution or radial diffusion assays (Zelezetsky and Tossi, 2006). The clinical potential of a given AMP

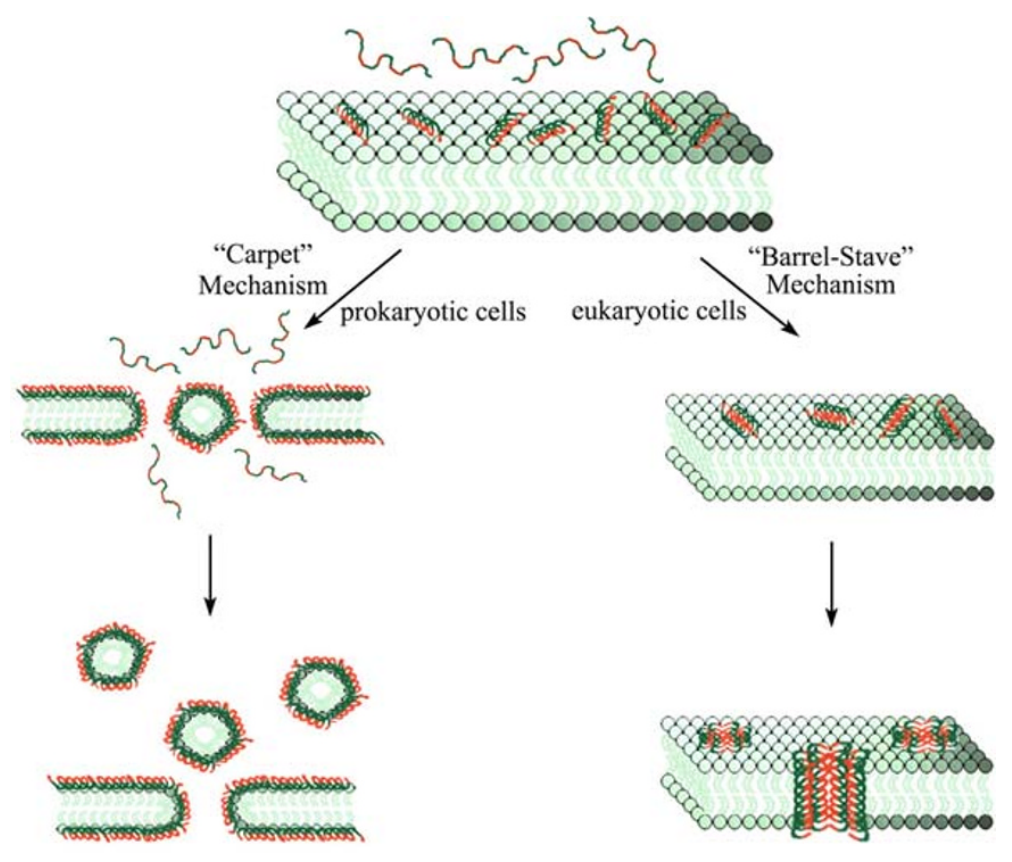

Figure 3. Membrane discrimination mechanism for cationic AMPs. The polar faces of helical peptides are colored in red and the non-polar faces of helical peptides are colored in green. 
is assessed by the ratio of $\mathrm{MHC}$ and MIC, or therapeutic index. Therapeutic index (MHC/MIC) can be improved by increasing the antimicrobial activity, decreasing hemolytic activity or both (Chen et al., 2005). High therapeutic index indicates low cytotoxic activity (high MHC value) but high antimicrobial activity (low MIC value).

In general, the antimicrobial activity and hemolytic activity in amphipathic aAMPs are related to multiple physicochemical parameters, including length, sequence, charge, helicity, hydrophobicity, amphipathicity, the hydrophobic/ hydrophilic angle and self-association. These parameters are also correlated, thus altering one will cause changes to the others, which makes it complicated to illuminate the influence of a single factor to the activity. We will review the current knowledge of the roles of these parameters on the activity together with the corresponding modification upon each parameter in Part I and present some specific ways to optimize activities of aAMPs in Part II.

\section{Part I}

Length

Natural aAMPs are generally small with $<50$ amino acid residues (Hancock, 1997). Considering the minimal requirements of 3-4 amino acid residues per turn in an alpha-helix, 7-8 amino acid residues (two turns) will be required for the formation of amphipathic faces. As for the transverse of lipid bilayers, at least 22 residues will be needed for aAMPs (Shai, 2002). The impact of size for aAMP activity has been intensively investigated.

Benincasa et al. reported that a 15-residue synthetic peptide corresponding to the C-terminus of melittin, had about 5-7 times less antimicrobial activity but 300 times less hemolytic activity compared to the original melittin (Benincasa et al., 2003). In another study, a potent antimicrobial peptide HP-A3 was synthesized by deleting the $\mathrm{N}$-terminal random coil of the $\alpha$-helical antimicrobial peptide HP (2-20). The derived 15 mer peptide HP-A3 (A3-NT) showed enhanced antibacterial and antifungal activity at 2- and 4-fold, respectively, without hemolysis (Park et al., 2007).

\section{Sequence}

Natural aAMPs have different primary sequences, with approximately $50 \%$ hydrophobic residues. Many of them have a hinge or 'kink' caused by proline or glycine residues. Tossi et al. compared the $\mathrm{N}$-terminal $\alpha$-helical domain $(20$ residues) of over 150 natural AMPs and did not find a conserved pattern. The positional residue conservation was very poor excepting position 1 (70\% Gly) and 8 (50\% Lys). However, the positional conservation of residue types, i.e., charged, neutral or hydrophobic, was presented in a relatively defined way (Tossi et al., 2000).

\section{Charge}

It is generally accepted that the interaction of AMPs with membrane is largely dependent on the electrostatic force. However, the correlation between peptide charge and biological activity is complicated. Alteration of peptide charge will inevitably cause changes to other parameters. Jiang et al. systematically altered the charges of the 26-residue amphipathic a-helical antimicrobial peptide $\mathrm{V} 13 \mathrm{~K}$ to analyze the impact of $\mathrm{V} 13 \mathrm{~K}$ polar face charge on its biological activity (Jiang et al., 2008). By introducing or substituting basic amino acids or negatively-charged amino acids to the polar face of $\mathrm{V} 13 \mathrm{~K}$, they demonstrated that both the number of positivelycharged residues on the polar face and the peptide net charge play important roles in antimicrobial activity and hemolytic activity of AMPs. The most dramatic observation is the sharp alteration of hemolytic activity by increasing a single positive charge from +8 to +9 on the polar face of V13K. For both antimicrobial activity and hemolytic activity, reducing the net charge to lower than +4 renders $V 13 \mathrm{~K}$ analogs totally inactive. However, increasing the net charge from +4 to +8 made $V 13 \mathrm{~K}$ analogs more active for antimicrobial activity, while maintaining low hemolytic activity (MHC $250 \mu \mathrm{g} / \mathrm{mL}$ ). Further increase in net charge to +9 and +10 improved antimicrobial activity, but also caused a dramatic increase in hemolytic activity. These data suggest that there is a critical threshold for the net positive charge or positive charge density on a given $\alpha$-helical AMP that governs antimicrobial and hemolytic activities (Jiang et al., 2008).

Helicity

Multiple studies demonstrated that peptide helicity might be more important for toxicity (neutral membrane) than antimicrobial activity (negatively charged membrane) of AMPs (Pouny et al., 1992; Dathe et al., 1996). The most convincing evidences are from D-amino acid substitution on model peptides. The enantiomer substitution does not alter other parameters, such as charge and hydrophobicity, but it does change the peptide structure as monitored by the reversedphase HPLC (Chen et al., 2002). The nonpolar face of an amphipathic $\alpha$-helical peptide represents a preferred domain for binding to the hydrophobic matrix of a reversed-phase column. The disturbance of helix by incorporation of D-amino acid will cause the change of overall hydrophobicity, which is reflected by the retention time, and the peptide hydrophobicity value is calculated by the side-chain hydrophobicity (Kovacs et al., 2006).

The disruption of helical structure by D-amino acid substitution in a hydrophobic environment depends on the number of substitutions and the original helical propensity. For example, substitution of four $\mathrm{D}$-amino acids into the melittin B sequences for their L-residues dramatically 
reduced a-helical structure in 40\% TFE, from 73\% ahelical content for melittin to $15 \%$ for the above D-analog (Oren and Shai, 1997). The advantage of D-amino acid substitutions is D-analog shows negligible cytotoxic effects on mammalian cells and retained the antibacterial activity, while the native melittin B is extremely hemolytic $(100 \%$ hemolysis at $1 \mu \mathrm{g} / \mathrm{mL}$ ). Chen et al. reported a systematic study to determine impacts of D-amino acid substitution in the nonpolar face of an amphipathic a-helical peptide on secondary structure (Chen et al., 2002). The results showed the feasibility of controlled disruption of helical structure by Damino acid substitution in aqueous medium, whist still allowing peptides to be fully folded as $\alpha$-helical structure in a hydrophobic environment. Therefore, D-amino acid substitution in an AMP may be an effective approach to dissociate the antimicrobial activity against cytotoxicity of eukaryotic cells.

Previous studies revealed that the incorporation of Damino acids into native and model non-selective cell lytic peptides with either $\alpha$-helix or $\beta$-sheet structures resulted in reduction of their cytotoxicity on mammalian cells, while the peptides retained their antibacterial properties (Shai and Oren, 1996; Avrahami et al., 2001). To determine the underlying mechanism, many studies analyzed the activities of diastereomers that possess an amphipathic structure, assuming that they play similar roles as native AMPs composed by L-amino acids only (Zasloff, 1987; Mor et al., 1991; Falla et al., 1996; Shai and Oren, 1996).

In an investigation to analyze the consequence of sequence alteration of an amphipathic $\alpha$-helical AMP and its diastereomers, the sequence of an amphipathic $\alpha$-helical peptide was altered, and the structure, function and interaction with model membranes and intact bacteria were compared to the diastereomers (Papo et al., 2002). The Lamino acid peptides are originally composed of leucine and lysine, whereas one-third of the sequence of the diastereomers consists of $\mathrm{D}$-amino acids. The $\mathrm{L}$-amino acid peptides and the diastereomers showed similar functions, although they had different structures in the membrane as revealed by attenuated total reflectance Fourier-transform infrared spectroscopy (ATR-FTIR). These results indicated that Ddiastereomers were non-hemolytic and maintained full activity in serum, whereas the L-amino acid peptides were highly hemolytic, with poor solubility and low activity in serum, and could be fully cleaved by trypsin and proteinase K. Thus, D-amino acid substitution prevented the diastereomers from enzymatic degradation. Transmembrane potential depolarization experiments in model membranes and intact bacteria indicate that the killing mechanism of the diastereomers depends on their ability to diffuse into the inner bacterial membrane. Taken together, these data reveal the advantage of the diastereomers over the L-amino acid counterparts as candidates for developing new antibiotics for systemic use.

\section{Hydrophobicity}

Although the precise mechanism of action of AMPs has not been fully deciphered, it is believed that the cytoplasmic membrane is the main target of AMPs and hydrophobicity is a crucial parameter for their biological activities. In general, increased hydrophobicity of the non-polar face of an amphipathic $\alpha$-helical peptide improves antimicrobial activity (Dathe et al., 1997; Wieprecht et al., 1997; Avrahami and Shai, 2002).

Lee et al. utilized the sequence of native peptide HP (2-20) and designed several analogs with amino acid substitutions to alter the net hydrophobicity. HP (2-20) (AKKVFKRLEKLFSKIQNDK) is the antimicrobial sequence derived from the $\mathrm{N}$-terminus of Helicobacter pylori ribosomal protein L1 (RPL1). In this study, the substitution of GIn and Asp at positions 17 and 19 of HP (2-20) with Trp enhanced hydrophobicity and also led to a dramatic increase in antimicrobial activity without alteration in hemolytic activity. In contrast, the reduction of hydrophobicity by substituting Leu and Phe at positions 12 and 19 of HP (2-20) with Ser, did not result in significant impacts on the antimicrobial activity. These results indicated that the hydrophobicity of peptides is critical for its effective antimicrobial activity and may be one of the determinant parameters for peptide interaction with the lipid bilayers of the cell membrane (Lee et al., 2002).

On the other hand, Chen et al. utilized a 26-residue amphipathic $\alpha$-helical antimicrobial peptide to analyze the impacts of peptide hydrophobicity on the action of AMPs, and found that there was an optimal hydrophobicity window in which high antimicrobial activity could be obtained (Chen et al., 2007). Decreased or increased hydrophobicity beyond this window dramatically inhibited antimicrobial activity, and higher hydrophobicity was also correlated with stronger hemolytic activity. It can be explained by the strong peptide self-association that prevents the peptide from passing through the cell wall in prokaryotic cells, whereas such peptide self-association does not affect the access to eukaryotic membranes.

\section{Amphipathicity and hydrophobic moment}

The ability to assume amphipathic structure is a functionally important requirement for AMP incorporation into membranes. Amphipathicity is usually quantitated by the hydrophobic moment defined as the vector sum of the hydrophobicity of individual amino acids (Eisenberg, 1984).

Several studies have shown that on the angle of helix content the mean hydrophobic moment is a more important factor affecting antimicrobial activity than hydrophobicity (Pathak et al., 1995; Fernandez-Vidal et al., 2007). The measurements of the interfacial partitioning of a family of 17residue amidated-acetylated peptides into both neutral and anionic lipid vesicles showed that peptide helicity in water and 
interface increased linearly with hydrophobic moment, as did the favorable peptide partitioning free energy. This results demonstrates that helical amphipathicity plays more significant roles in interfacial binding than hydrophobicity does (Fernandez-Vidal et al., 2007).

Angle subtended by the hydrophilic/hydrophobic faces on the wheel projection

In an ideal helical peptide, the angle sustained by polar and nonpolar facets can be clearly exhibited using a helix wheel projection. It is proposed that the angle influences the location of the peptide within the membrane and the structure of the transmembrane pores (Dathe and Wieprecht, 1999).

\section{Self-association}

Previous studies have shown that peptide self-association (i. e., the ability to dimerize) in aqueous solutions is a important parameter for antimicrobial activity (Chen et al., 2005). If the self-association ability of a peptide is too strong in aqueous medium, it could decrease the ability of the peptide to dissociate, to pass through the capsule and cell wall of microorganisms, and to penetrate into the cytoplasmic membrane to kill target cells. The major components of the capsule are high-molecular-weight polysaccharides. The cell wall of gram-negative bacteria includes the outer membrane, which contains proteins, lipopolysaccharides, porins and lipids of the bilayer, and the peptidoglycan layer between the outer and inner (cytoplasmic) membranes. The exact components of the cell wall that restrict the access of the folded peptide into the cytoplasmic membrane remain unknown. The self-association ability of peptides is commonly determined by RP-HPLC temperature profiling technique (Mant et al., 2003).

All together, these physico-chemical parameters of aAMPs are intimately related. Alteration of one parameter commonly leads to changes to other parameters, and research groups take different approaches to their investigations. Furthermore, some studies are based on an ideal helix, but the helicity of peptides, even when induced by the interaction with membrane, is always less than $100 \%$. So design or modification of AMPs based on the helical wheel projection together with these parameters often neglects the actual structure difference from the desired helix. In addition to the general parameters as discussed above, there are still some key factors to impact the specificity of aAMPs.

\section{Part II}

Amidation of the C-terminal carboxyl increases antimicrobial activity

It has been shown that amidation of the C-terminal carboxyl group is able to improve the antimicrobial activity of AMPs
(Falla et al., 1996; Oren and Shai, 1996; Jia et al., 2000; Konno et al., 2007). When the structures of the AMP dermaseptin S3 and its amidated analog were compared, Shalev et al. found that the amidated dermaseptin has an induced and/or stabilized $\alpha$-helical conformation, and thus, is more rigid and extended than the nonamidated analog (Shalev et al., 2002). Circular dichroism measurements suggested that the peptides form an a-helical structure in aqueous solution. However, NMR measurements revealed that native dermaseptin S3 does not contain a-helical elements, while the amidated analog shows a single fourresidue helix. The native peptide contains a hydrogenbonded turn and bend, and is more flexible, while the amidated analog has a defined a-helix at the C-terminal region, and is significantly elongated and more rigid in terms of structure. Therefore, amidation not only modifies the net positive charge, but also stabilizes peptide structure and improves antimicrobial activity.

All D-form of aAMPs increase antimicrobial activity and stability

It is hypothesized that cell membrane receptors would require a specific peptide chirality to be active if the peptide relys upon a ligand-receptor mechanism to kill bacteria. Enantiomeric forms of AMPs with D-amino acids only were used to investigate this membrane-binding mechanism (Wade et al., 1990; De Lucca et al., 2000; Bland et al., 2001). However, many studies illustrated that $\mathrm{D}$-amino acid peptides have equal activities with their L-enantiomers (Wade et al., 1990; Hong et al., 1999; Wakabayashi et al., 1999; De Lucca et al., 2000; Bland et al., 2001; Hamamoto et al., 2002; Elmquist and Langel, 2003), suggesting that the antimicrobial mechanism of these peptides does not involve a stereoselective interaction with a chiral enzyme, lipid or protein receptor. However, some data indicate that the D-form AMPs have enhanced antimicrobial activity in vitro and in vivo due to higher stability against proteolysis degradation, which suggests higher clinical therapeutic potential (Vunnam et al., 1998; Chen et al., 2006; Jung et al., 2007).

Substitution of a single positively charged residue in the center of the non-polar face of amphipathic AMPs to reduce hemolysis

To determine the relationship of structure and mechanism of action of amphipathic $\alpha$-helical AMPs, Chen et al. introduced single positively-charged amino acid substitution in the center of non-polar face (position 13) on the helix $V_{681}$ peptide, which is a 26-residue peptide resulting from semi-random DNA mutagenesis of an analog of a Cecropin A (1-8) Melittin B (1-18) hybrid. (Chen et al., 2005). $V_{681}$ peptide has strong hemolytic activity and high antimicrobial activity against Gram-negative and Gram-positive bacteria (Zhang et al., 
1998; Zhang et al., 1999). Compared to $V_{681}$, the Lys substitution analog $\mathrm{V} 13 \mathrm{~K}$ has the highest therapeutic index (MHC/MIC) against Gram-negative and Gram-positive bacteria due to coupled reduction of hemolytic activity and antimicrobial activity. Disruption of the hydrophobic surface with the Lys substitution $\left(\mathrm{V} 13 \mathrm{~K}_{\mathrm{L}}\right)$ would prevent dimerization of the peptide in aqueous solution, allowing the peptide to enter the membrane interface region more effectively. At the same time, the alteration of the hydrophobic surface also prevented deeper penetration of the peptide into the hydrophobic core of the membrane, and thus, the hemolytic activity of peptide was minimized.

Selective fluorination of peptides enhanced chemical and thermal stability with increased hydrophobicity

Meng et al. demonstrated that fluorinated derivatives of two AMPs, buforin and magainin, show similar or enhanced antibacterial activity and improved protease stability (Meng and Kumar, 2007). It is interesting that hemolytic activity of peptides in the buforin series was negligible while fluorinated magainin analogues displayed increased hemolysis compared to the parent peptides. These results suggest that fluorination of AMPs may be an effective approach to improve the peptide stability against proteolysis degradation.

\section{CONCLUSIONS}

aAMPs utilize various mechanisms different from conventional antibiotics and provide an enormous advantage, including broad-spectrum activity, rapid killing and low bacterial resistance. Thus, it is proposed that these peptides have high potential for clinical application as antimicrobial agents in the near future. Many studies have been performed in order to identify aAMPs with high antimicrobial activity and low cytotoxicity against human cells. Peptide scientists are discovering and designing more AMPs from natural sources and the laboratory. The precise mechanism of action of AMPs remains to be elucidated. The era of peptides as antibiotics will come soon when more peptide molecules with an optimal therapeutic index enter clinical practice.

\section{ACKNOWLEDGEMENTS}

This work was supported by Natural Science Foundation of China Grant No. 30840029, Grants for Doctorate Program of New Teacher, Ministry of Education of China, No. 20070183062, No. 200801831064 and the Excellent Youth Program of the Jilin Provincial Science \& Technology Department, China, Grant No. 20070111.

\section{ABBREVIATIONS}

AMP, antimicrobial peptides; aAMPs, $\alpha$-helical antimicrobial peptides; ATR-FTIR, attenuated total reflectance Fourier-transform infrared spectroscopy; CL, cardiolipin; MD, molecular dynamics; MHC, minimal hemolytic concentration; MIC, minimal inhibitory concentration; PC, phosphatidylcholine; PE, phosphatidylethanolamine; PG, phosphatidylglycerol; PS, phosphatidylserine; RPL1, ribosomal protein L1; SM, sphingomyelin

\section{REFERENCES}

Abraham, T., Lewis, R.N., Hodges, R.S., and McElhaney, R.N. (2005). Isothermal titration calorimetry studies of the binding of the antimicrobial peptide gramicidin $S$ to phospholipid bilayer membranes. Biochemistry 44, 11279-11285.

Ali, F.E., Cao, G., Poudyal, A., Vaara, T., Nation, R.L., Vaara, M., and $\mathrm{Li}$, J. (2009). Pharmacokinetics of novel antimicrobial cationic peptides NAB 7061 and NAB 739 in rats following intravenous administration. J Antimicrob Chemother 64, 1067-1070.

Avrahami, D., Oren, Z., and Shai, Y. (2001). Effect of multiple aliphatic amino acids substitutions on the structure, function, and mode of action of diastereomeric membrane active peptides. Biochemistry 40, 12591-12603.

Avrahami, D., and Shai, Y. (2002). Conjugation of a magainin analogue with lipophilic acids controls hydrophobicity, solution assembly, and cell selectivity. Biochemistry 41, 2254-2263.

Benincasa, M., Skerlavaj, B., Gennaro, R., Pellegrini, A., and Zanetti, M. (2003). In vitro and in vivo antimicrobial activity of two alphahelical cathelicidin peptides and of their synthetic analogs. Peptides 24, 1723-1731.

Bland, J.M., De Lucca, A.J., Jacks, T.J., and Vigo, C.B. (2001). All-Dcecropin B: Synthesis, conformation, lipopolysaccharide binding, and antibacterial activity. Mol Cell Biochem 218, 105-111.

Brogden, K.A. (2005). Antimicrobial peptides: pore formers or metabolic inhibitors in bacteria? Nat Rev 3, 238-250.

Bryan, L.E. (1988). General Mechanisms of Resistance to Antibiotics. $J$ Antimicrob Chemoth 22, 1-15.

Chen, Y., Guarnieri, M.T., Vasil, A.I., Vasil, M.L., Mant, C.T., and Hodges, R.S. (2007). Role of peptide hydrophobicity in the mechanism of action of alpha-helical antimicrobial peptides. Antimicrob Agents Chemother 51, 1398-1406.

Chen, Y., Mant, C.T., Farmer, S.W., Hancock, R.E., Vasil, M.L., and Hodges, R.S. (2005). Rational design of alpha-helical antimicrobial peptides with enhanced activities and specificity/therapeutic index. J Biol Chem 280, 12316-12329.

Chen, Y., Mant, C.T., and Hodges, R.S. (2002). Determination of stereochemistry stability coefficients of amino acid side-chains in an amphipathic alpha-helix. J Pept Res 59, 18-33.

Chen, Y.X., Vasil, A.I., Rehaume, L., Mant, C.T., Burns, J.L., Vasil, M. L., Hancock, R.E.W., and Hodges, R.S. (2006). Comparison of biophysical and biologic properties of alpha-helical enantiomeric antimicrobial peptides. Chem Biol Drug Des 67, 162-173.

Dathe, M., Schumann, M., Wieprecht, T., Winkler, A., Beyermann, M., Krause, E., Matsuzaki, K., Murase, O., and Bienert, M. (1996). Peptide helicity and membrane surface charge modulate the balance of electrostatic and hydrophobic interactions with lipid bilayers and biological membranes. Biochemistry 35, 12612-12622.

Dathe, M., and Wieprecht, T. (1999). Structural features of helical antimicrobial peptides: their potential to modulate activity on model membranes and biological cells. Bba-Biomembranes 1462, 71-87.

Dathe, M., Wieprecht, T., Nikolenko, H., Handel, L., Maloy, W.L., 
MacDonald, D.L., Beyermann, M., and Bienert, M. (1997). Hydrophobicity, hydrophobic moment and angle subtended by charged residues modulate antibacterial and haemolytic activity of amphipathic helical peptides. Febs Lett 403, 208-212.

De Lucca, A.J., Bland, J.M., Vigo, C.B., Jacks, T.J., Peter, J., and Walsh, T.J. (2000). D-Cecropin B: proteolytic resistance, lethality for pathogenic fungi and binding properties. Med Mycol 38, 301-308.

Ehrenstein, G., and Lecar, H. (1977). Electrically gated ionic channels in lipid bilayers. Q Rev Biophys 10, 1-34.

Eisenberg, D. (1984). Three-dimensional structure of membrane and surface proteins. Annu Rev Biochem 53, 595-623.

Elmquist, A., and Langel, U. (2003). In vitro uptake and stability study of pVEC and its all-D analog. Biol Chem 384, 387-393.

Falla, T.J., Karunaratne, D.N., and Hancock, R.E.W. (1996). Mode of action of the antimicrobial peptide indolicidin. J Biol Chem 271, 19298-19303.

Fernandez-Vidal, M., Jayasinghe, S., Ladokhin, A.S., and White, S.H. (2007). Folding amphipathic helices into membranes: amphiphilicity trumps hydrophobicity. J Mol Biol 370, 459-470.

Giuliani, A., Pirri, G., and Nicoletto, S.F. (2007). Antimicrobial peptides: an overview of a promising class of therapeutics. Cent Eur J Biol 2, 1-33.

Hallock, K.J., Lee, D.K., and Ramamoorthy, A. (2003). MSI-78, an analogue of the magainin antimicrobial peptides, disrupts lipid bilayer structure via positive curvature strain. Biophys J 84, 3052-3060.

Hamamoto, K., Kida, Y., Zhang, Y., Shimizu, T., and Kuwano, K. (2002). Antimicrobial activity and stability to proteolysis of small linear cationic peptides with D-amino acid substitutions. Microbiol Immunol 46, 741-749.

Hancock, R.E. (1997). Peptide antibiotics. Lancet 349, 418- 422.

Hancock, R.E., and Rozek, A. (2002). Role of membranes in the activities of antimicrobial cationic peptides. FEMS Microbiol Lett 206, 143-149.

Hong, S.Y., Oh, J.E., and Lee, K.H. (1999). Effect of D-amino acid substitution on the stability, the secondary structure, and the activity of membrane-active peptide. Biochem Pharmacol 58, 1775-1780.

Jenssen, H., Hamill, P., and Hancock, R.E. (2006). Peptide antimicrobial agents. Clin Microbiol Rev 19, 491-511.

Jia, X., Patrzykat, A., Devlin, R.H., Ackerman, P.A., Iwama, G.K., and Hancock, R.E.W. (2000). Antimicrobial peptides protect coho salmon from Vibrio anguillarum infections. Appl Environ Microb 66, 1928-1932.

Jiang, Z.Q., Vasil, A.I., Hale, J.D., Hancock, R.E.W., Vasil, M.L., and Hodges, R.S. (2008). Effects of net charge and the number of positively charged residues on the biological activity of amphipathic alpha-helical cationic antimicrobial peptides. Biopolymers 90, 369-383.

Joanne, P., Falord, M., Chesneau, O., Lacombe, C., Castano, S., Desbat, B., Auvynet, C., Nicolas, P., Msadek, T., and El Amri, C. (2009). Comparative study of two plasticins: specificity, interfacial behavior, and bactericidal activity. Biochemistry 48, 9372-9383.

Jung, H.J., Park, Y., Sung, W.S., Suh, B.K., Lee, J., Hahm, K.S., and Lee, D.G. (2007). Fungicidal effect of pleurocidin by membraneactive mechanism and design of enantiomeric analogue for proteolytic resistance. Biochim Biophys Acta 1768, 1400-1405.
Konno, K., Rangel, M., Oliveira, J.S., Dos Santos Cabrera, M.P., Fontana, R., Hirata, I.Y., Hide, I., Nakata, Y., Mori, K., Kawano, M., et al. (2007). Decoralin, a novel linear cationic alpha-helical peptide from the venom of the solitary eumenine wasp Oreumenes decoratus. Peptides 28, 2320-2327.

Kovacs, J.M., Mant, C.T., and Hodges, R.S. (2006). Determination of intrinsic hydrophilicity/hydrophobicity of amino acid side chains in peptides in the absence of nearest-neighbor or comformational effects. Biopolymers 84, 283-297.

Ladokhin, A.S., and White, S.H. (2001). 'Detergent-like' permeabilization of anionic lipid vesicles by melittin. Biochim Biophys Acta 1514, 253-260.

Lee, D.G., Kim, H.N., Park, Y.K., Kim, H.K., Choi, B.H., Choi, C.H., and Hahm, K.S. (2002). Design of novel analogue peptides with potent antibiotic activity based on the antimicrobial peptide, HP (220), derived from $\mathrm{N}$-terminus of Helicobacter pylori ribosomal protein L1. Bba-Proteins Proteom 1598, 185-194.

Leontiadou, H., Mark, A.E., and Marrink, S.J. (2006). Antimicrobial peptides in action. J Am Chem Soc 128, 12156-12161.

Lugtenberg, B., and Van Alphen, L. (1983). Molecular architecture and functioning of the outer membrane of Escherichia coli and other gram-negative bacteria. Biochim Biophys Acta 737, 51-115.

Manna, M., and Mukhopadhyay, C. (2009). Cause and effect of melittin-induced pore formation: a computational approach. Langmuir 25, 12235-12242.

Mant, C.T., Chen, Y., and Hodges, R.S. (2003). Temperature profiling of polypeptides in reversed-phase liquid chromatography - I. Monitoring of dimerization and unfolding of amphipathic alphahelical peptides. J Chromatogr A 1009, 29-43.

Marr, A.K., Gooderham, W.J., and Hancock, R.E. (2006). Antibacterial peptides for therapeutic use: obstacles and realistic outlook. Curr Opin Pharmacol 6, 468-472.

Marshall, S.H., and Arenas, G. (2003). Antimicrobial peptides: A natural alternative to chemical antibiotics and a potential for applied biotechnology. Electron J Biotechn 6, 271-284.

Matsuzaki, K. (1999). Why and how are peptide-lipid interactions utilized for self-defense? Magainins and tachyplesins as archetypes. Biochim Biophys Acta 1462, 1-10.

Matsuzaki, K., Murase, O., Fujii, N., and Miyajima, K. (1995). Translocation of a channel-forming antimicrobial peptide, magainin 2 , across lipid bilayers by forming a pore. Biochemistry 34 , 6521-6526.

Matsuzaki, K., Murase, O., Fujii, N., and Miyajima, K. (1996). An antimicrobial peptide, magainin 2, induced rapid flip-flop of phospholipids coupled with pore formation and peptide translocation. Biochemistry 35, 11361-11368.

Mavri, J., and Vogel, H.J. (1996). Ion pair formation of phosphorylated amino acids and lysine and arginine side chains: a theoretical study. Proteins 24, 495-501.

Meng, H., and Kumar, K. (2007). Antimicrobial activity and protease stability of peptides containing fluorinated amino acids. J Am Chem Soc 129, 15615-15622.

Mor, A., Nguyen, V.H., Delfour, A., Miglioresamour, D., and Nicolas, P. (1991). Isolation, Amino-Acid-Sequence, and Synthesis of Dermaseptin, a Novel Antimicrobial Peptide of Amphibian Skin. Biochemistry 30, 8824-8830.

Neu, H.C. (1992). The crisis in antibiotic resistance. Science 257, 1064-1073. 
Oren, Z., and Shai, Y. (1996). A class of highly potent antibacterial peptides derived from pardaxin, a pore-forming peptide isolated from Moses sole fish Pardachirus marmoratus. Eur J Biochem 237, 303-310.

Oren, Z., and Shai, Y. (1997). Selective lysis of bacteria but not mammalian cells by diastereomers of melittin: Structure-function study. Biochemistry 36, 1826-1835.

Oyston, P.C., Fox, M.A., Richards, S.J., and Clark, G.C. (2009). Novel peptide therapeutics for treatment of infections. J Med Microbiol 58, 977-987.

Papo, N., Oren, Z., Pag, U., Sahl, H.G., and Shai, Y. (2002). The consequence of sequence alteration of an amphipathic alphahelical antimicrobial peptide and its diastereomers. J Biol Chem 277, 33913-33921.

Park, Y., Park, S.C., Park, H.K., Shin, S.Y., Kim, Y., and Hahm, K.S. (2007). Structure-activity relationship of $\mathrm{HP}(2-20)$ analog peptide: Enhanced antimicrobial activity by $\mathrm{N}$-terminal random coil region deletion. Biopolymers 88, 199-207.

Pathak, N., Salasauvert, R., Ruche, G., Janna, M.H., Mccarthy, D., and Harrison, R.G. (1995). Comparison of the Effects of Hydrophobicity, Amphiphilicity, and Alpha-Helicity on the Activities of Antimicrobial Peptides. Proteins 22, 182-186.

Pouny, Y., Rapaport, D., Mor, A., Nicolas, P., and Shai, Y. (1992). Interaction of antimicrobial dermaseptin and its fluorescently labeled analogues with phospholipid membranes. Biochemistry 31, 12416-12423.

Powers, J.P., and Hancock, R.E. (2003). The relationship between peptide structure and antibacterial activity. Peptides 24, 1681-1691.

Resende, J.M., Moraes, C.M., Munhoz, V.H., Aisenbrey, C., Verly, R. M., Bertani, P., Cesar, A., Pilo-Veloso, D., and Bechinger, B. (2009). Membrane structure and conformational changes of the antibiotic heterodimeric peptide distinctin by solid-state NMR spectroscopy. Proc Natl Acad Sci U S A 106, 16639-16644.

Shai, Y. (1999). Mechanism of the binding, insertion and destabilization of phospholipid bilayer membranes by alpha-helical antimicrobial and cell non-selective membrane-lytic peptides. Biochim Biophys Acta 1462, 55-70.

Shai, Y. (2002). Mode of action of membrane active antimicrobial peptides. Biopolymers 66, 236-248.

Shai, Y., and Oren, Z. (1996). Diastereomers of cytolysins, a novel class of potent antibacterial peptides. J Biol Chem 271, 7305-7308.

Shalev, D.E., Mor, A., and Kustanovich, I. (2002). Structural consequences of carboxyamidation of dermaseptin S3. Biochemistry $41,7312-7317$.

Tossi, A., Sandri, L., and Giangaspero, A. (2000). Amphipathic, alphahelical antimicrobial peptides. Biopolymers 55, 4-30.

Tytler, E.M., Anantharamaiah, G.M., Walker, D.E., Mishra, V.K., Palgunachari, M.N., and Segrest, J.P. (1995). Molecular basis for prokaryotic specificity of magainin-induced lysis. Biochemistry 34 , 4393-4401.
Verdon, J., Falge, M., Maier, E., Bruhn, H., Steinert, M., Faber, C., Benz, R., and Hechard, Y. (2009). Detergent-like activity and alpha-helical structure of warnericin RK, an anti-legionella peptide. Biophysical J 97, 1933-1940.

Vunnam, S., Juvvadi, P., Rotondi, K.S., and Merrifield, R.B. (1998). Synthesis and study of normal, enantio, retro, and retroenantio isomers of cecropin A-melittin hybrids, their end group effects and selective enzyme inactivation. J Pept Res 51, 38-44.

Wade, D., Boman, A., Wahlin, B., Drain, C.M., Andreu, D., Boman, H. G., and Merrifield, R.B. (1990). All-D Amino Acid-Containing Channel-Forming Antibiotic Peptides. Proc Natl Acad Sci U S A 87, 4761-4765.

Wakabayashi, H., Matsumoto, H., Hashimoto, K., Teraguchi, S., Takase, M., and Hayasawa, H. (1999). N-acylated and D enantiomer derivatives of a nonamer core peptide of lactoferricin B showing improved antimicrobial activity. Antimicrob Agents Ch 43, 1267-1269.

Wang, G., Li, X., and Wang, Z. (2009). APD2: the updated antimicrobial peptide database and its application in peptide design. Nucleic Acids Res 37, D933-937.

Wieprecht, T., Dathe, M., Beyermann, M., Krause, E., Maloy, W.L., MacDonald, D.L., and Bienert, M. (1997). Peptide hydrophobicity controls the activity and selectivity of magainin 2 amide in interaction with membranes. Biochemistry 36, 6124-6132.

Wildman, K.A.H., Lee, D.K., and Ramamoorthy, A. (2003). Mechanism of lipid bilayer disruption by the human antimicrobial peptide, LL-37. Biochemistry 42, 6545-6558.

Wu, M.H., Maier, E., Benz, R., and Hancock, R.E.W. (1999). Mechanism of interaction of different classes of cationic antimicrobial peptides with planar bilayers and with the cytoplasmic membrane of Escherichia coli. Biochemistry 38, 7235-7242.

Yeaman, M.R., and Yount, N.Y. (2003). Mechanisms of antimicrobial peptide action and resistance. Pharmacological Rev 55, 27-55.

Zasloff, M. (1987). Magainins, a Class of Antimicrobial Peptides from Xenopus Skin - Isolation, Characterization of 2 Active Forms, and Partial Cdna Sequence of a Precursor. Proc Natl Acad Sci U S A 84, 5449-5453.

Zelezetsky, I., and Tossi, A. (2006). Alpha-helical antimicrobial peptides-using a sequence template to guide structure-activity relationship studies. Biochim Biophys Acta 1758, 1436-1449.

Zhang, L., Benz, R., and Hancock, R.E. (1999). Influence of proline residues on the antibacterial and synergistic activities of alphahelical peptides. Biochemistry 38, 8102-8111.

Zhang, L., Falla, T., Wu, M., Fidai, S., Burian, J., Kay, W., and Hancock, R.E. (1998). Determinants of recombinant production of antimicrobial cationic peptides and creation of peptide variants in bacteria. Biochem Biophys Res Commun 247, $674-680$.

Zilberstein, D., Schuldiner, S., and Padan, E. (1979). Proton electrochemical gradient in Escherichia coli cells and its relation to active transport of lactose. Biochemistry 18, 669-673. 